\title{
Singular behaviour near the tip of a sharp V-notch in a power law hardening material
}

\author{
LIN XIA and TZUCHIANG WANG \\ LNM, Institute of Mechanics, Chinese Academy of Sciences, Beijing, 100080, People's Republic of China
}

Received 20 February 1991; accepted in revised form 16 March 1992

\begin{abstract}
This paper presents an asymptotic analysis of the near-tip stress and strain fields of a sharp V-notch in a power law hardening material. First, the asymptotic solutions of the HRR type are obtained for the plane stress problem under symmetric loading. It is found that the angular distribution function of the radial stress $\sigma_{r}$ presents rapid variation with the polar angle if the notch angle $\beta$ is smaller than a critical notch angle; otherwise, there is no such phenomena. Secondly, the asymptotic solutions are developed for antisymmetric loading in the cases of plane strain and plane stress. The accurate calculation results and the detailed comparisons are given as well. All results show that the singular exponent $s$ is changeable for various combinations of loading condition and plane problem.
\end{abstract}

\section{Introduction}

In 1968, Hutchinson [3, 4], Rice and Rosengren [5] published their well-known asymptotic analyses on the stress and strain fields near the tip of a crack in a power law hardening material. They obtained the separable singular stress and strain fields, now known as 'HRR singular solutions'. Following their work, Kuang and $\mathrm{Xu}$ [6] presented an asymptotic analysis for a sharp V-notch of a tensile plate in 1987, and found some principles in which the singular stresses and strains change along with notch angle. They only researched the plane strain problem (herein designated as PSN), however, under symmetric load.

However, from the work of Hutchinson [3, 4], we can find that the radial stress $\sigma_{r}$ varies rapidly within a certain polar angular region in plane stress problem (PSS) of mode I. This rapid variation makes the calculation much more difficult compared with PSN. Thus, it is natural to ask if there is the same behaviour in the case of a sharp V-notch. The present paper will be able to answer this problem. That is to say, further investigations will be made in detail on PSS's stress and strain singular fields near the tip of a sharp V-notch under symmetric loading. The detailed comparison between our results and PSN's results [6] will be discussed.

The stress and strain fields near the tip of a sharp V-notch under antisymmetric loading are also developed systematically in both PNS and PSS. As a result, the complete stress and strain fields near the tip of a sharp V-notch are obtained for all combinations of loading condition and plane problem.

In Williams [1], the stress singularities at the vertex of an elastic sector plate under extension were investigated in detail, where vertex angle runs from 0 to $360^{\circ}$. For the elastic opening crack problem Carpenter [7] found two eigenvector solutions which give stress singularities as $r \rightarrow 0$. His first solution corresponds to our mode I solution; and another one corresponds to our mode II solution for $n=1$. 


\section{Basic equations}

Following Hutchinson [3], the power law hardening material's deformation obeys the Ramberg-Osgood formula

$$
\varepsilon=\sigma+\alpha \sigma^{n}
$$

where $\sigma$ is the nondimensional stress, $\sigma=\bar{\sigma} / E, \bar{\sigma}$ is dimensional stress, $E$ is Young's elastic modulus, $n$ and $\alpha$ are hardening exponent and hardening coefficient respectively.

Throughout this paper, all quantities without bar ' $\because$ ' will be nondimensional ones, unless otherwise stated.

Comparing with the plastic strains, the elastic strains in (2.1) are small, and can be neglected in asymptotic analysis. Hence, according to the plastic deformation theory, the three dimensional stress-strain relations take the form

$$
\varepsilon_{i j}=\frac{3}{2} \alpha \sigma_{e}^{n-1} S_{i j}
$$

where $\sigma_{e}$ is the effective stress, defined as $\sigma_{e}^{2}=\frac{3}{2} S_{i j} S_{i j}, S_{i j}$ is the stress deviator: $S_{i j}=\sigma_{i j}$ $\frac{1}{3} \sigma_{k k} \delta_{i j}$.

Equilibrium can be guaranteed provided that the stress functions are introduced:

$$
\left\{\begin{array}{l}
\sigma_{r}=\frac{1}{r}\left(\frac{\partial \phi}{\partial r}+\frac{1}{r} \frac{\partial^{2} \phi}{\partial \theta^{2}}\right), \\
\sigma_{\theta}=\frac{\partial^{2} \phi}{\partial r^{2}} \\
\tau_{r \theta}=-\frac{\partial}{\partial r}\left(\frac{1}{r} \frac{\partial \phi}{\partial \theta}\right),
\end{array}\right.
$$

where $\phi=\bar{\phi} / E L, \bar{\phi}$ is the dimensional stress function, and $\phi$ is the nondimensional one; $r=\bar{r} / L$, $L$ is a characteristic length which is taken as the depth of notch, as Fig. 1 shows.

The strain compatibility equation is

$$
\frac{1}{r} \frac{\partial^{2}}{\partial r^{2}}\left(r \varepsilon_{\theta}\right)+\frac{1}{r^{2}} \frac{\partial^{2}}{\partial \theta^{2}} \varepsilon_{r}-\frac{1}{r} \frac{\partial}{\partial r} \varepsilon_{r}-\frac{2}{r^{2}} \frac{\partial^{2}}{\partial r \partial \theta}\left(r \varepsilon_{r \theta}\right)=0
$$

The boundary conditions:

(i) For symmetric loading:

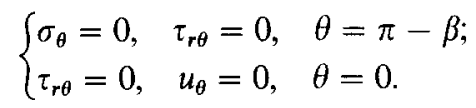




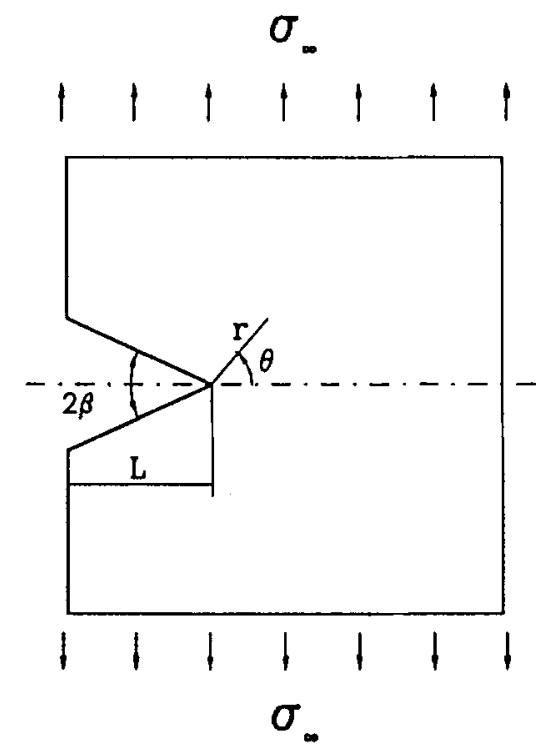

Fig. 1(a). Symmetric loading.

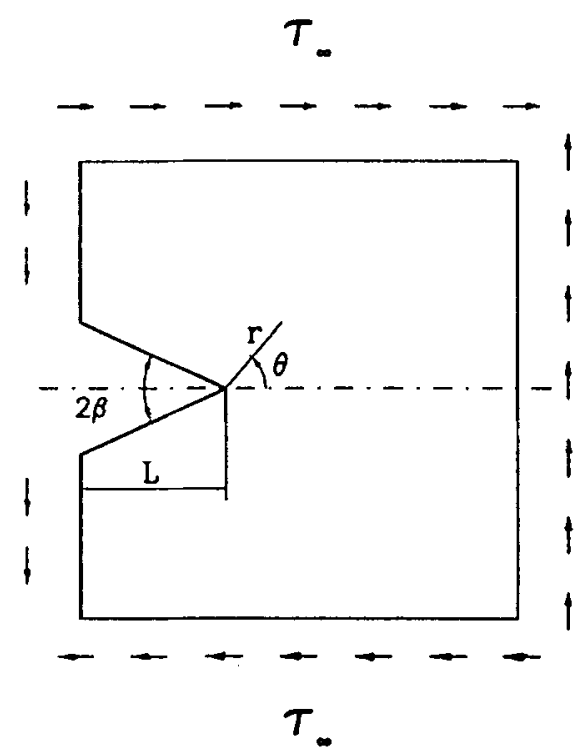

Fig. I(b). Antisymmetric loading.

(ii) For antisymmetric loading:

$$
\begin{cases}\sigma_{\theta}=0, & \tau_{r \theta}=0, \quad \theta=\pi-\beta \\ \sigma_{\theta}=0, & u_{r}=0, \quad \theta=0\end{cases}
$$

The equations from (2.2) to (2.6) constitute the governing equations for our problems.

\section{Analysis method}

Assuming

$$
\phi=K r^{s+2} \tilde{\phi}(\theta)
$$

we have

$$
\left\{\begin{array}{l}
\sigma_{r}=K r^{s} \tilde{\sigma}_{r}(\theta) \\
\sigma_{\theta}=K r^{s} \tilde{\sigma}_{\theta}(\theta) \\
\tau_{r \theta}=K r^{s} \tilde{\tau}_{r \theta}(\theta)
\end{array}\right.
$$

where

$$
\left\{\begin{array}{l}
\tilde{\sigma}_{r}(\theta)=\tilde{\phi}^{\prime \prime}+(s+2) \tilde{\phi}(\theta), \\
\tilde{\sigma}_{\theta}(\theta)=(s+2)(s+1) \tilde{\phi}(\theta), \\
\tilde{\tau}_{r \theta}(\theta)=-(s+1) \tilde{\phi}^{\prime}(\theta) .
\end{array}\right.
$$


The strains are

$$
\left\{\begin{array}{l}
\varepsilon_{r}=\alpha K^{n} r^{n s} \tilde{\varepsilon}_{r}(\theta), \\
\varepsilon_{\theta}=\alpha K^{n} r^{n s} \tilde{\varepsilon}_{\theta}(\theta), \\
\varepsilon_{r \theta}=\alpha K^{n} r^{n s} \widetilde{\varepsilon}_{r \theta}(\theta),
\end{array}\right.
$$

where $\tilde{\sigma}_{e}(\theta)$ and $\tilde{\varepsilon}_{i j}(\theta)$ are divided into two forms for different plane problems

(1) PSS (plane stress):

$$
\left\{\begin{array}{l}
\tilde{\sigma}_{e}^{2}=\tilde{\sigma}_{r}^{2}+\tilde{\sigma}_{\theta}^{2}-\tilde{\sigma}_{r} \tilde{\sigma}_{\theta}+3 \tilde{\tau}_{r \theta}^{2}, \\
\tilde{\varepsilon}_{r}(\theta)=\tilde{\sigma}_{e}^{n-1}\left(\tilde{\sigma}_{r}-\frac{\tilde{\sigma}_{\theta}}{2}\right), \\
\tilde{\varepsilon}_{\theta}(\theta)=\tilde{\sigma}_{e}^{n-1}\left(\tilde{\sigma}_{\theta}-\frac{\tilde{\sigma}_{r}}{2}\right), \\
\tilde{\varepsilon}_{r \theta}(\theta)=\frac{3}{2} \tilde{\sigma}_{e}^{n-1} \tilde{\tau}_{r \theta},
\end{array}\right.
$$

(2) PSN (plane strain):

$$
\begin{aligned}
& \tilde{\sigma}_{e}^{2}=\frac{3}{4}\left(\tilde{\sigma}_{r}-\tilde{\sigma}_{\theta}\right)^{2}+3 \tilde{\tau}_{r \theta}^{2}, \\
& \left\{\begin{array}{l}
\tilde{\varepsilon}_{r}(\theta)=-\tilde{\varepsilon}_{\theta}(\theta)=\frac{3}{4} \tilde{\sigma}_{e}^{n-1}\left(\tilde{\sigma}_{r}-\tilde{\sigma}_{\theta}\right), \\
\tilde{\varepsilon}_{r \theta}(\theta)=\frac{3}{2} \tilde{\sigma}_{e}^{n-1} \tilde{\tau}_{r \theta} .
\end{array}\right.
\end{aligned}
$$

Substituting (3.4) into (2.4), we obtain

$$
\tilde{\varepsilon}_{r}^{\prime \prime}+n s(n s+1) \tilde{\varepsilon}_{\theta}-n s \tilde{\varepsilon}_{r}-2(n s+1) \tilde{\varepsilon}_{r \theta}^{\prime}=0 .
$$

Using $\mathcal{\phi}(\theta)$, the boundary conditions can be represented as:

(i) For symmetric loading:

$$
\left\{\begin{array}{l}
\tilde{\phi}^{\prime}(0)=\tilde{\phi}^{\prime \prime \prime}(0)=0, \\
\tilde{\phi}(\pi-\beta)=\tilde{\phi}^{\prime}(\pi-\beta)=0 .
\end{array}\right.
$$

(ii) For antisymmetric loading:

$$
\left\{\begin{array}{l}
\tilde{\phi}(0)=\tilde{\phi}^{\prime \prime}(0)=0 \\
\tilde{\phi}(\pi-\beta)=\tilde{\phi}^{\prime}(\pi-\beta)=0 .
\end{array}\right.
$$

The homogeneous differential equation (3.9), together with homogeneous boundary conditions (3.10) or (3.11), define a two-point boundary value problem. Equivalently, it can be viewed as a nonlinear eigenvalue problem where the singular exponent $s$ is the eigenvalue. The shooting method is used to solve this problem, and the correspondent initial value problem is integrated by means of a Runge-Kutta method in which each integrating step length can be adjusted 
automatically as expected. During the solving procedure, the numerical accuracy of each integration step and the accuracy within which the boundary conditions are met, can also be controlled. In principle, we are able to obtain any high accuracy solution. In practical calculating performance, all calculations are controlled within the accuracy of $10^{-8}$ in this paper.

\section{The singular stress and strain fields}

Case 1. PSS (plane stress) under symmetrical loading.

If $n=1$, the compatibility equation becomes

$$
\tilde{\phi}^{\prime \prime \prime \prime}(\theta)+\left[(s+2)^{2}+s^{2}\right] \tilde{\phi}^{\prime \prime}(\theta)+(s+2)^{2} s^{2} \tilde{\phi}(\theta)=0
$$

which is the same as the one of the PSN problem under the same loading condition. Thus, it can be concluded that the variation pattern of singular exponent $s$ versus the notch angle $\beta$ is the same as that of PSN. According to [2], $s$ can be determined using the follow equation

$$
\sin (2 \omega) /(2 \omega)+\sin [2(s+1) \omega] /[2(s+1) \omega]=0,
$$

where $\omega=\pi-\beta$.

If $n>1$, the issues will be completely different.

Some eigenvalues $s$ for various $\beta$ and $n$ are calculated, and then tabulated into Table 1 and Table 2, respectively.

Table 1 shows that the eigenvalue of PSS isn't equal to one of PSN in general for a fixed $\beta$, and the absolute value $|s|$ of PSS is slightly larger than one of PSN. But when $\beta$ equals zero degree, the values $s$ of two stress conditions become the same, just like one defined by the $J$-integral: $s=-1 /(n+1)$. Table 2 shows the variation of $s$ vs. $n$ at $\beta=60^{\circ}$. Generally speaking, $|s|$ of PSS is larger than the correspondent one of PSN.

During the calculation, we find it is difficult to find an exact solution as $\beta$ is approximately equal to $23.5^{\circ}$ for $n=3$. The reason lies in the fact that $\tilde{\phi}_{\max }^{\prime \prime \prime}$ approaches infinity at such a notch

Table 1

\begin{tabular}{llllllll}
\hline \multicolumn{7}{c}{ Singular exponent $s$ for $n=3.00$} \\
\hline$\beta$ & $0^{\circ}$ & $15^{\circ}$ & $30^{\circ}$ & $45^{\circ}$ & $60^{\circ}$ & $75^{\circ}$ & $90^{\circ}$ \\
\hline PSN & -0.25000 & -0.24802 & -0.24115 & -0.22509 & -0.19248 & -0.12926 & 0.00000 \\
PSS & -0.25000 & -0.24920 & -0.24897 & -0.23546 & -0.20081 & -0.13386 & 0.00000 \\
\hline
\end{tabular}

\begin{tabular}{|c|c|c|c|c|}
\hline \multirow[b]{2}{*}{$n$} & \multicolumn{2}{|c|}{ Singular exponent $s$} & \multicolumn{2}{|c|}{$\beta=60^{\circ}$} \\
\hline & 1.00 & 3.00 & 9.00 & 13.00 \\
\hline PSN & -0.38427 & -0.19248 & -0.08261 & -0.06066 \\
\hline PSS & -0.38427 & -0.20081 & -0.09082 & -0.06767 \\
\hline
\end{tabular}

Table 2 
angle. This feature can be clearly seen in Fig. 2 . The angular distribution function $\tilde{\sigma}_{r}(\theta)$ of the radial stress $\sigma_{r}$ has the rapid variation region if $\beta<23.5^{\circ}$. For $\beta>23.5^{\circ}$, the angular distribution $\tilde{\sigma}_{r}(\theta)$ varies smoothly without any rapid variation region. In order to discuss conveniently, we define such a critical transition notch angle as $\beta_{\mathrm{cr}}$, that is to say, as $\beta \rightarrow \beta_{\mathrm{cr}}$, $\tilde{\phi}_{\max }^{\prime \prime \prime} \rightarrow-\infty$.

The angular distributions of all strain components with $n=3$ for $\beta=0^{\circ}, 15^{\circ}, 30^{\circ}, 45^{\circ}, 60^{\circ}$ and $75^{\circ}$ are presented in Fig. 3.

It is clear that the strain components versus $\beta$ vary rapidly compared with the stress components. Among them, the variation of $\varepsilon_{r}$ versus $\beta$ is most distinct.

The angular distribution of stresses at $\beta=60^{\circ}$ for $n=1,3,9,13$ are plotted in Fig. 4. It can be seen that the plots of stresses versus $n$ show a weak dependence on $n$. On the contrary, the variation of strains versus $n$ is remarkable, as shown in Fig. 5.

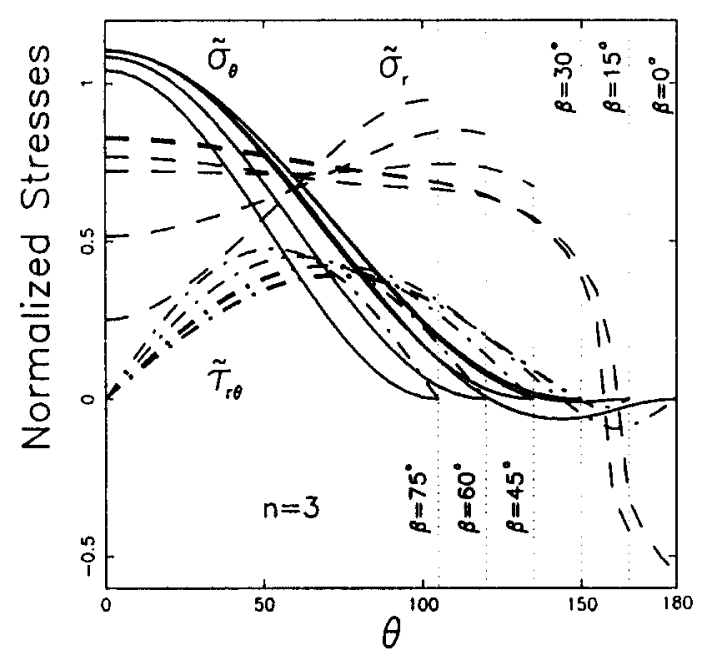

Fig. 2. $\theta$-variations for normalized stresses at $n=3$ for $\beta=0^{\circ}, 15^{\circ}, 30^{\circ}, 45^{\circ}, 60^{\circ}, 75^{\circ}$ (PSS, symmetric loading).

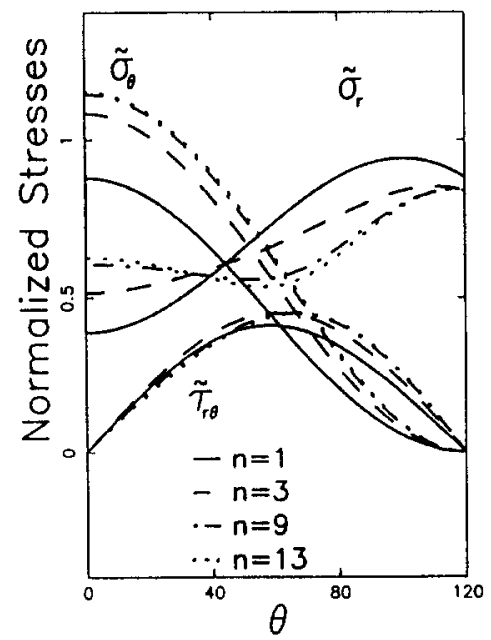

Fig. 4. $\theta$-variations for normalized stresses at $\theta=60^{\circ}$ for $n=1,3,9,13$ (PSS symmetric loading).

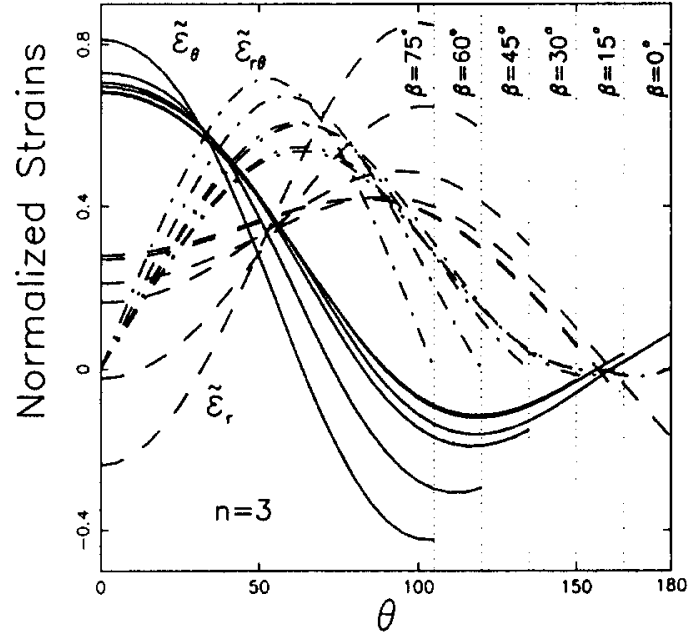

Fig. 3. $\theta$-variations for normalized strains at $n=3$ for $\beta=0^{\circ}, 15^{\circ}, 30^{\circ}, 45^{\circ}, 60^{\circ}, 75^{\circ}$ (PSS, symmetric loading).

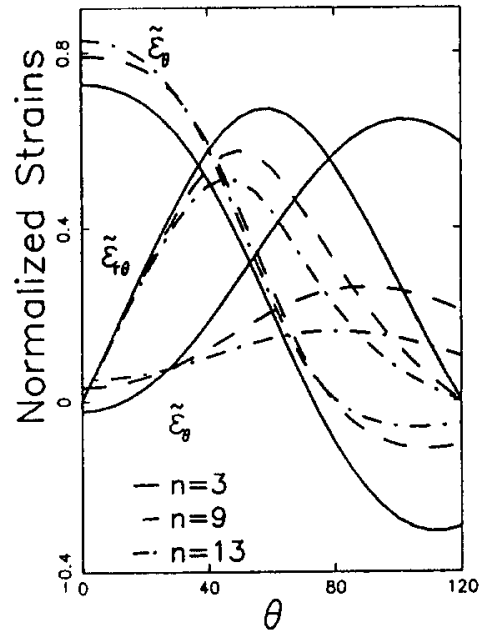

Fig. 5. $\theta$-variations for normalized strains at $\beta=60^{\circ}$ for $n=3,9,13$ (PSS, symmetric loading). 
Table 3

\begin{tabular}{lllll}
\hline$\theta=0^{\circ}$ & & & & $n=3$ \\
\hline & $\beta$ & $0^{\circ}$ & $45^{\circ}$ & $75^{\circ}$ \\
\hline \multirow{2}{*}{ PSN } & $\sigma_{\theta} / \sigma_{e}$ & 7.59671 & 2.49172 & 1.44927 \\
& $\sigma_{m} / \sigma_{e}$ & 7.01936 & 1.91437 & 0.87192 \\
\hline \multirow{2}{*}{ PSS } & $\sigma_{\theta} / \sigma_{e}$ & 1.10607 & 1.10563 & 1.04162 \\
& $\sigma_{m} / \sigma_{e}$ & 0.62531 & 0.61011 & 0.43069 \\
\hline
\end{tabular}

Note: $\sigma_{m}=\left(\sigma_{r}+\sigma_{\theta}+\sigma_{z}\right) / 3$

Table 3 gives some values of the ratios $\sigma_{\theta}(0) / \sigma_{m}(0), \sigma_{m}(0) / \sigma_{e}(0)$ under both PSN and PSS for $n=3$. One can find that both the ratio $\sigma_{\theta}(0) / \sigma_{e}(0)$ and the ratio $\sigma_{m}(0) / \sigma_{e}(0)$ of PSN are all larger than those of PSS. It is concluded that the effects of triaxiality is much stronger in PSN. But when $\beta$ increases, the differences will gradually decrease.

Case 2. PSN (plane strain) and PSS (plane stress) under antisymmetric loading.

If $n=1$, namely elastic materials, the compatibility equation is the same as (4.1). And singular exponent $s$ can be evaluated from the following

$$
\sin (2 \omega) /(2 \omega)-\sin [2(s+1) \omega] /[2(s+1) \omega]=0,
$$

where $\omega=\pi-\beta$. It is noted that the above equation (4.3), is not only suitable to PSN but also suitable to PSS.

Clearly, under antisymmetric loading $s$ varies with $\beta$ in a different way from that of symmetric loading. Yet, this feature has not received enough attention.

Some evaluated eigenvalues $s$ for different $n$ and different $\beta$ have been listed in Table 4. From this table we can see that $s=-1 /(n+1)$ if $\beta=0$ (i.e. crack), exactly like the cases of symmetric loading. But if $\beta \neq 0, s$ is not identical for both PSN and PSS. Yet this difference is very small. Furthermore, it is hard to say whether or not $s$ of PSN is larger than of PSS. On the contrary, as mentioned above, the $|s|$ of PSS is always slightly larger than one of PSN under symmetric loading.

Table 4

\begin{tabular}{llllllc}
\hline \multicolumn{7}{c}{ Eigenvalue $s$} \\
\hline \multirow{2}{*}{1.00} & $\beta$ & $0^{\circ}$ & $15^{\circ}$ & $30^{\circ}$ & $45^{\circ}$ & $60^{\circ}$ \\
& psn & -0.50000 & -0.40181 & -0.26910 & -0.09147 & 0.0 \\
\hline \multirow{2}{*}{3.00} & pss & -0.50000 & -0.40181 & -0.26910 & -0.09147 & 0.0 \\
& psn & -0.25000 & -0.21385 & -0.16028 & -0.08013 & 0.0 \\
\hline \multirow{2}{*}{9.00} & pss & -0.25000 & -0.21450 & -0.15922 & -0.07362 & 0.0 \\
& psn & -0.10000 & -0.09262 & -0.07982 & -0.05661 & -0.01041 \\
\multirow{2}{*}{13.00} & pss & -0.10000 & -0.09294 & -0.07980 & -0.05490 & 0.0 \\
& psn & -0.07143 & -0.06747 & -0.06026 & -0.04628 & -0.01371 \\
& pss & -0.07143 & -0.06769 & -0.06038 & -0.04498 & -0.00835 \\
\hline
\end{tabular}




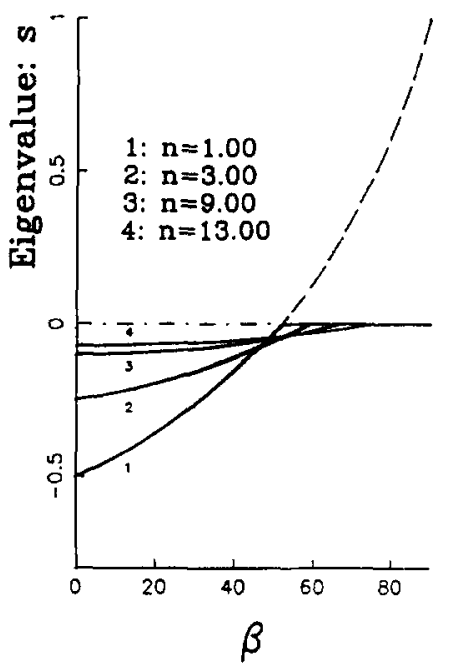

Fig. 6. $\beta$-variations of eigenvalues for $n=1,3,9,13$ under PSN condition and antisymmetric loading.

Figure 6 is plotted according to the data of PSN in Table 4. The figure clearly suggests that $s$ will change its sign from negative to positive as $\beta$ increases to a certain angle $\left(<90^{\circ}\right)$, and the corresponding notch angle, across which $s$ changes its sign, will gradually move to the right in the horizontal axis when $n$ increases. The alteration of the sign of $s$ means that singular behaviors of stresses will disappear. Actually the asymptotic stress and strain fields will in turn be controlled by the elastic stress and strain fields. It is worth noting that $s=0$ is always a root of (4.3) for elastic material. Therefore, as $s$ changes its sign from negative to positive, $s$ should not take the positive value given by the dash line in Fig. 6 but should always equals 0 . This issue is completely different from that of symmetric loading. In the latter case, there always exist singularities of stresses and strains within the whole range of $0<\beta<90^{\circ}$.

All figures from Figs. 7-9 belong to the solutions of stresses and strains of PSN. Among them, Fig. 7(a) and Fig. 7(b) show the stresses and strains respectively for $\beta=0^{\circ}$ (crack). And Fig. 8(a)

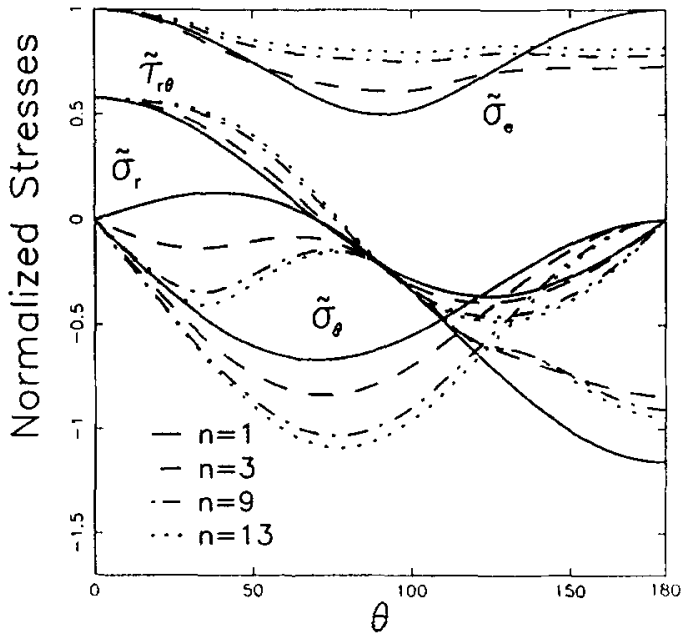

Fig. $7(a) . \theta$-variations for normalized stresses at $\beta=0^{\circ}$ for $n=1,3,9,13$ (PSN, antisymmetric loading).

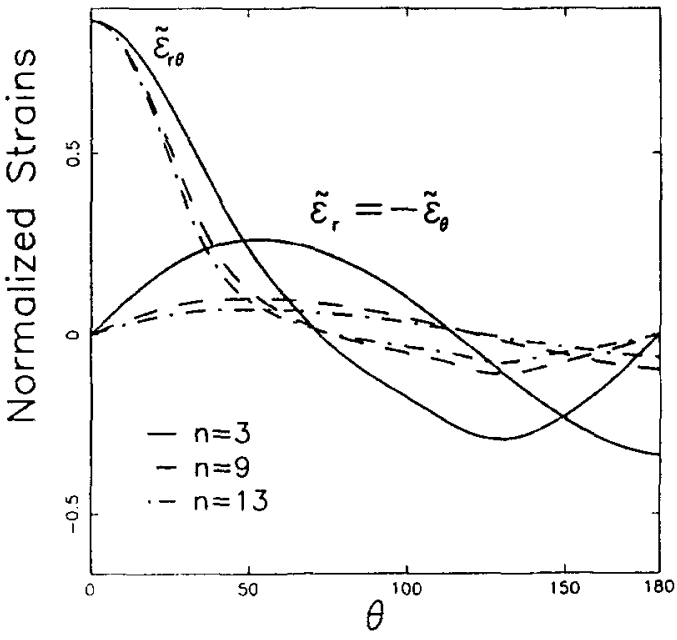

Fig. $7(b)$. $\theta$-variations for normalized strains at $\beta=0^{\circ}$ for $n=3,9,13$ (PSN, antisymmetric loading). 


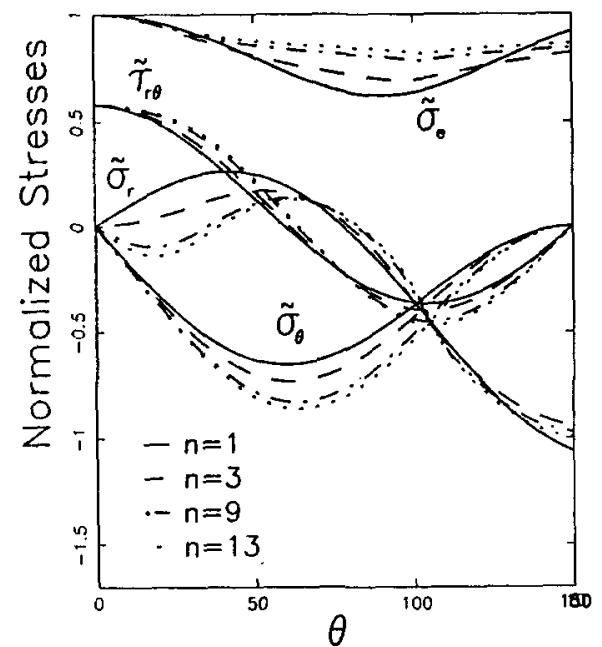

Fig. $8(a)$. $\theta$-variations for normalized stresses at $\beta=30^{\circ}$ for $n=1,3,9,13$ (PSN, antisymmetric loading).

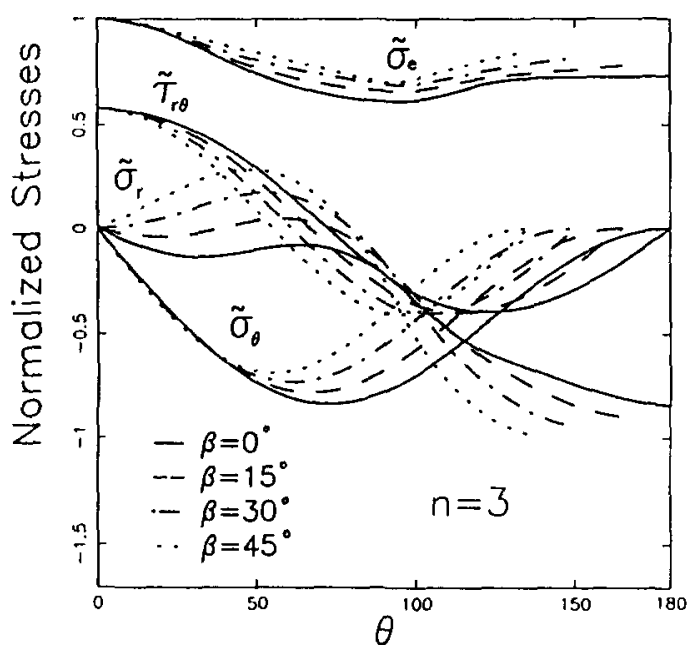

Fig. $9(a)$. $\theta$-variations for normalized stresses at $n=3$ for $\beta=0^{\circ}, 15^{\circ}, 30^{\circ}, 45^{\circ}$. (PSN, antisymmetric loading).

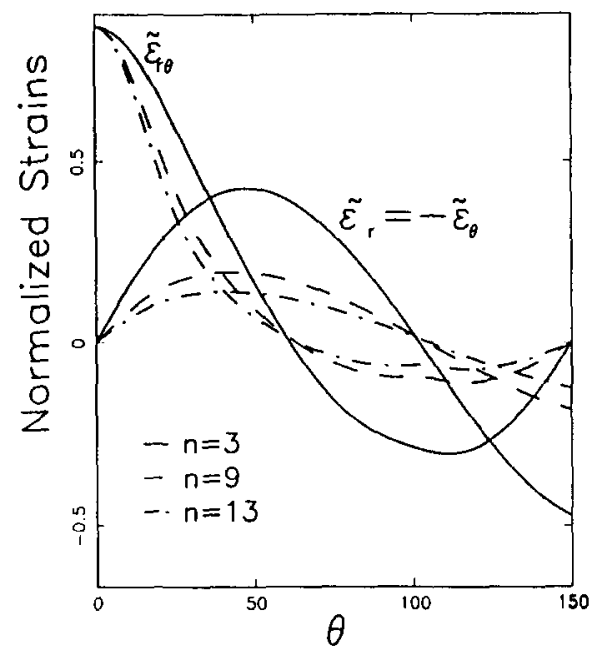

Fig. $8(b)$. $\theta$-variations for normalized strains at $\beta=30^{\circ}$ for $n=3,9,13$ (PSN, antisymmetric loading).

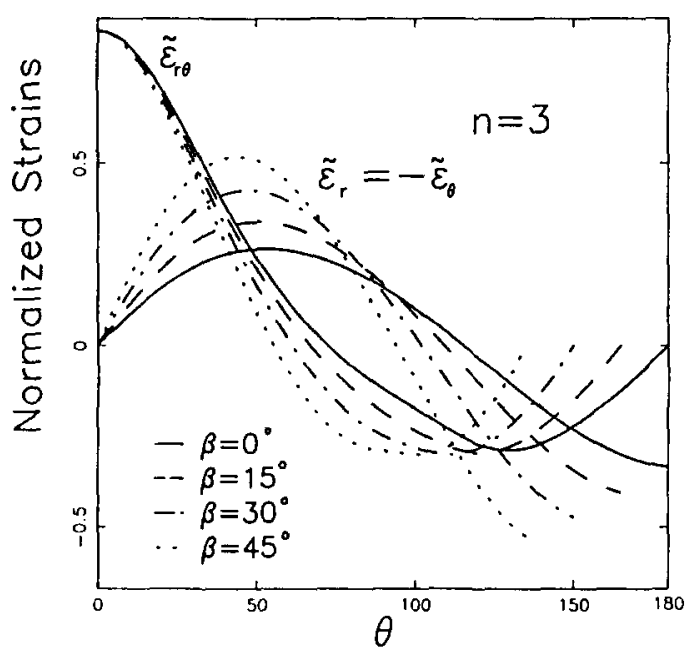

Fig. $9($ b) . $\theta$-variations for normalized strains at $n=3$ for $\beta=0^{\circ}, 15^{\circ}, 30^{\circ}, 45^{\circ}$. (PSN, antisymmetric loading).

and Fig. 8(b) present the stresses and strains respectively for $\beta=30^{\circ}$. From these figures, we can find that the stresses and strains will tend to weakly depend on $n$ as $n$ increases. Figures $9(\mathrm{a})$ and (9b) describe the stresses and strains respectively with $n=3$ for $\beta=10^{\circ}, 15^{\circ}, 30^{\circ}, 45^{\circ}$.

On the other hand, all figures from Figs 10-12 correspond to the solutions of stresses and strains of PSS.

First, comparing the solutions of PSS with ones of PSN, we can see that both results are quite close to each other, contrary to the situation of symmetric loading.

Next, Fig. 10(b) indicates that all strain components approach zero for about $\theta>80^{\circ}$ when $n$ is larger than 9. But as $\beta$ increases, this approaching trend becomes slower, as shown in Fig. 11(b).

Finally, among the cases of PSS under antisymmetric loading there aren't any rapid variation phenomena of $\tilde{\sigma}_{r}(\theta)$, contrary to that under symmetric loading. 


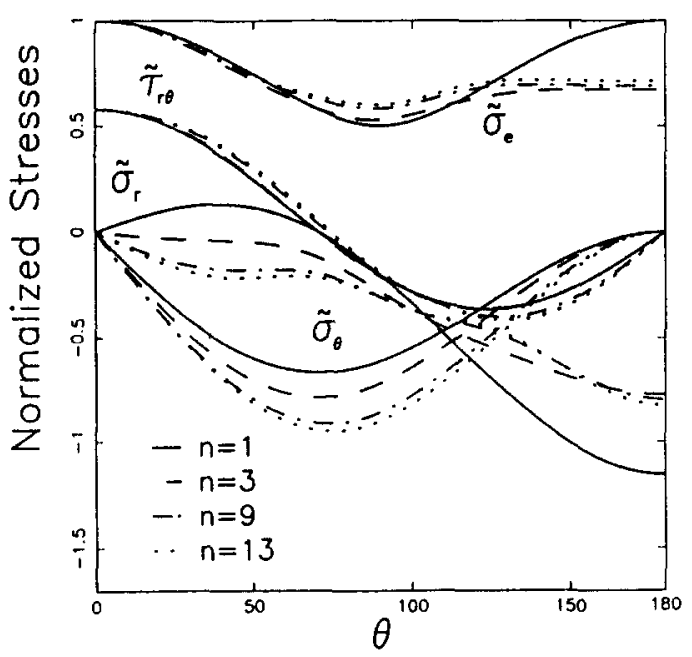

Fig. $10(a)$. $\theta$-variations for normalized stresses at $\beta=0^{\circ}$ for $n=1,3,9,13$. (PSS, antisymmetric loading).

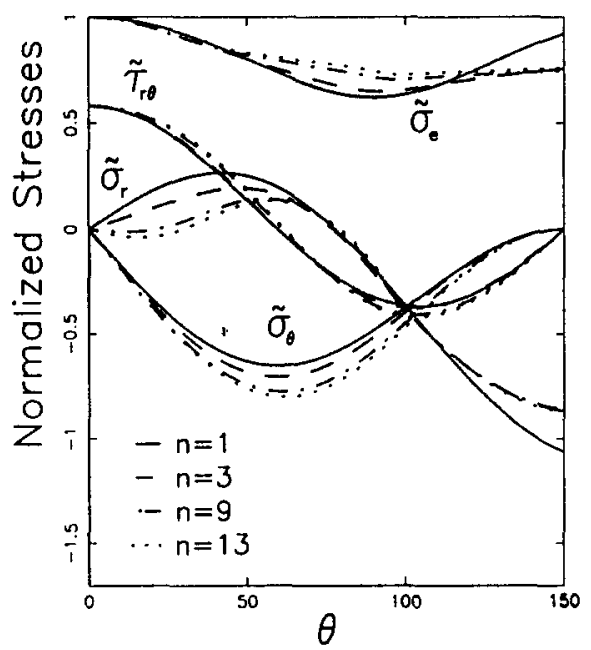

Fig. $11\left(\right.$ a). $\theta$-variations for normalized stresses at $\beta=30^{\circ}$ for $n=1,3,9,13$ (PSS, antisymmetric loading).

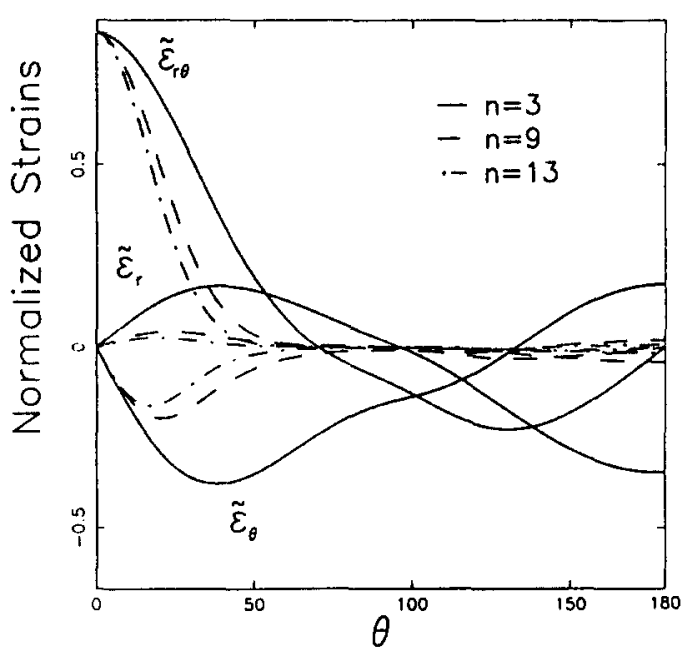

Fig. $10(b)$. $\theta$-variations for normalized strains at $\beta=0^{\circ}$ for $n=3,9,13$ (PSS, antisymmetric loading).

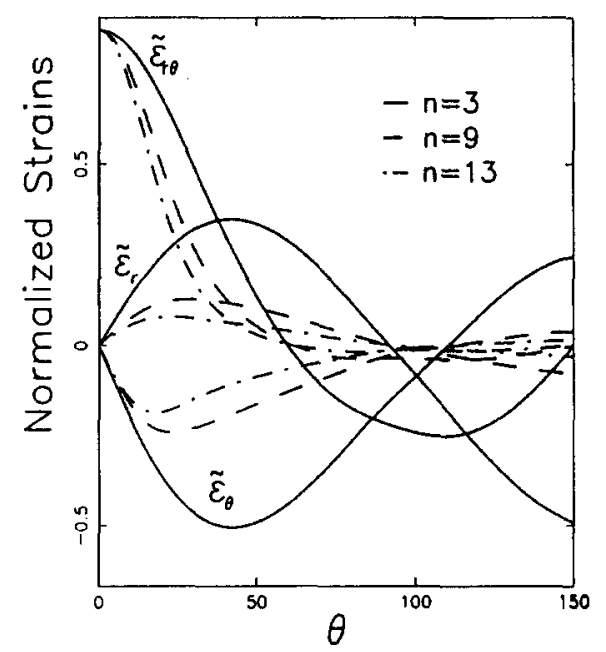

Fig. $11(b)$. $\theta$-variations for normalized strains at $\beta=30^{\circ}$ for $n=3,9,13$ (PSS, antisymmetric loading).

\section{Summary}

(1) As for elastic materials $(n=1)$, singular exponents $s$ of PSS and PSN are identical at any fixed $\beta$ under the same loading condition. Yet, the $s$ under symmetric loading differs from the $s$ under antisymmetric loading at any fixed $\beta$ for the same plane problem.

(2) If $\beta=0$ (crack), $s=-1 /(n+1)$ for any combination of loading stress conditions, as defined by the $J$-integral.

(3) If $n>1$, the corresponding singular exponents aren't equal to each other for both loading conditions. Under symmetric loading condition, singularities of stresses always exist within the whole region: $0^{\circ}<\beta<90^{\circ}$. But under antisymmetric loading condition, the singularities will disappear and become $s=0$ when $\beta$ increases and reaches a certain notch angle $\left(<90^{\circ}\right)$, 


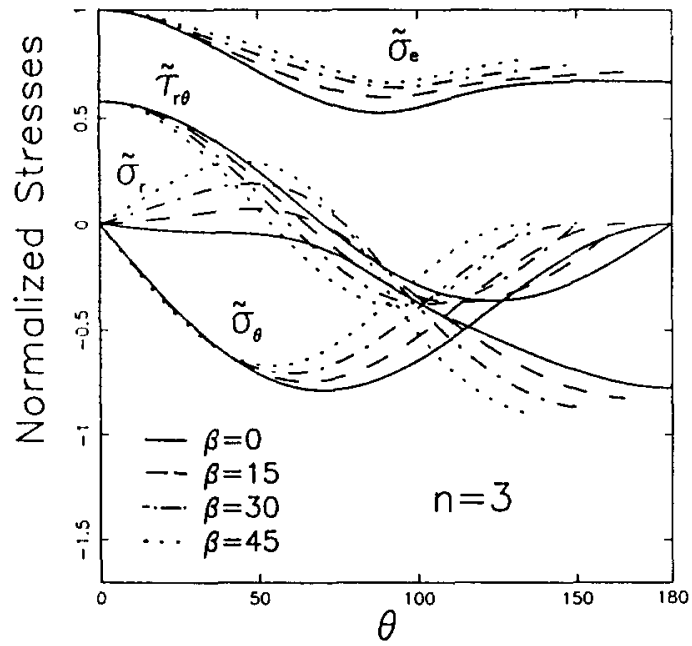

Fig. 12(a). $\theta$-variations for normalized stresses at $n=3$ for $\beta=0^{\circ}, 15^{\circ}, 30^{\circ}, 45^{\circ}$. (PSS, antisymmetric loading).

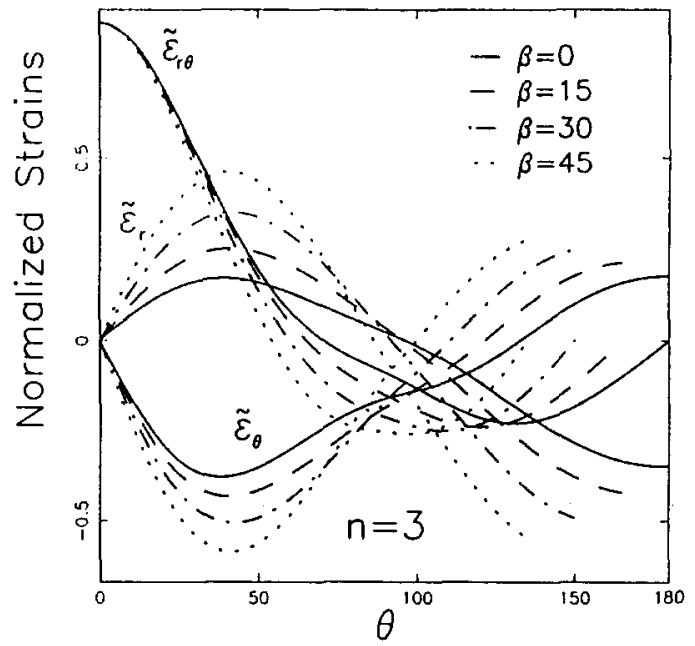

Fig. 12(b). $\theta$-variations for normalized strains at $n=3$ for $\beta=0^{\circ}, 15^{\circ}, 30^{\circ}, 45^{\circ}$. (PSS, antisymmetric loading).

which isn't the same for various $n$. Generally speaking, the notch angle, across which the sign of singular exponent $s$ alters, will shift to the right along the abscissa if $n$ increases.

(4) When $n>1$ and the loading conditions are fixed, there is no distinct difference between the value of the singular exponent $s$ of PSN and one of PSS.

(5) As for the combination of PSS and symmetric loading, a critical notch angle $\beta_{\mathrm{cr}}$ exists. If $\beta<\beta_{\mathrm{cr}}$, the angular distributions of $\tilde{\sigma}_{r}(\theta)$ versus $\theta$ have rapid variation behaviour, otherwise if $\beta>\beta_{\mathrm{cr}}$, there isn't such behaviour.

\section{Acknowledgements}

This project is supported by the National Science Foundation of China. Helpful discussions with Professor Yong-li Wu are gratefully acknowledged.

\section{References}

1. M.L. Williams, Journal of Applied Mechanics 19 (1952) 526-528.

2. S.N. Karp and F.C. Karal, Communication on Pure and Applied Mathematics XV (1962) 413-421.

3. J.W. Hutchinson, Journal of the Mechanics and Physics of Solids 16 (1968a) 13-31.

4. Ibid, 16 (1968b) 332-347.

5. J.R. Rice and G.F. Rosengren, Journal of the Mechanics and Physics of Solids 16 (1968) 1-12.

6. Z.B. Kuang and X. P. Xu, International of Fracture 35 (1987) 39-53.

7. W.C. Carpenter, International Journal of Fracture 26 (1984) 201-214. 